\title{
mRNA COVID vaccine and myocarditis in adolescents
}

This article was published on 16 Aug 2021 at www.hkmj.org.

Mike YW Kwan ${ }^{1 \#, ~ M S c ~(A p p l i e d ~ E p i d e m i o l o g y) ~(C U H K), ~ F H K A M ~(P a e d i a t r i c s), ~ G i l b e r t ~ T ~ C h u a 2 \#, ~ M B, ~ B S, ~ F H K A M ~(P a e d i a t r i c s), ~}$ CB Chow ${ }^{1,2 \#, ~ M D, ~ F H K A M ~(P a e d i a t r i c s), ~ S a b r i n a ~ S L ~ T s a o ², ~ M B, ~ B S(U K), ~ F A C C, ~ K e l v i n ~ K W ~ T o ³, ~ M D, ~ F R C P a t h, ~}$ KY Yuen ${ }^{*}$, MD, FRCPath, YL Lau *, MD (Hon), FRCPCH, Patrick Ip ${ }^{2}$ *, MPH, FHKAM (Paediatrics)

${ }^{1}$ Department of Paediatrics and Adolescent Medicine, Princess Margaret Hospital, Hong Kong

${ }^{2}$ Department of Paediatrics and Adolescent Medicine, Li Ka Shing Faculty of Medicine, The University of Hong Kong, Hong Kong

${ }^{3}$ Department of Microbiology, Carol Yu Centre for Infection, Li Ka Shing Faculty of Medicine, The University of Hong Kong, Hong Kong

\author{
\# These authors contributed equally to the work. \\ *Corresponding authors: kyyuen@hku.hk, lauylung@hku.hk, patricip@hku.hk \\ Hong Kong Med J 2021;27:326-7 \\ https://doi.org/10.12809/hkmj215120
}

In Hong Kong, the coronavirus disease 2019 (COVID-19) vaccination programme started on 26 February 2021. CoronaVac (an inactivated virus vaccine developed by Sinovac) and Comirnaty (BNT162b2 mRNA vaccine co-developed by BioNTech and Pfizer, and manufactured and distributed in China by Fosun Pharma) are the available formulations for public use. Comirnaty is safe and provides good antibody response, including for patients aged 12 to 15 years with clinical efficacy in protecting against severe acute respiratory syndrome coronavirus 2 (SARS-CoV-2) infection of $100 \% .{ }^{1}$ On 14 June 2021, the Food and Health Bureau, Hong Kong SAR Government lowered the age limit for receiving the Comirnaty vaccine to $\geq 12$ years. $^{2}$

Since April 2021, there have been reports that myocarditis and pericarditis occur more frequently in adolescents and young adults after mRNA COVID-19 vaccinations internationally. ${ }^{3-5}$ In Hong Kong, Comirnaty is the only mRNA technology platform COVID-19 vaccine available, but others are available elsewhere (eg, from Moderna).

Myocarditis and pericarditis have many virological and immunological causes, and are known to occur after vaccination; for example, the incidence of myocarditis after smallpox vaccination is around 12 to 16.1 per million vaccinated individuals. Myocarditis can also occur after SARS-CoV-2 infection alone or as a consequence of multisystem inflammatory syndrome in children after COVID-19 or paediatric inflammatory multisystem syndrome temporally associated with SARS-CoV-2.,7,8

Reported cases of myocarditis after mRNA COVID-19 vaccination are mostly male adolescents and young adults aged between 12 and 24 years who received the second dose of the mRNA vaccine. ${ }^{9-14}$ These heart complications are exceedingly rare, but are increasingly reported as hundreds of million doses of mRNA COVID-19 vaccines have been administered worldwide. ${ }^{4,5,9,10}$ The typical symptoms of myocarditis and pericarditis are chest pain, shortness of breath, and palpitations occurring within 1 week (usually 2-4 days) after vaccination. The condition is mild in most of the affected individuals, with only minimal treatment required and full recovery within a few days.

Although myocarditis and pericarditis have many virological and immunological causes, a causal link was suspected due to the immunological reaction to the mRNA COVID-19 vaccine. Individuals are recommended to rest and refrain from heavy strenuous activities for 1 week after mRNA COVID-19 vaccination, which will be helpful during the rare occurrence of myocarditis or pericarditis. Individuals experiencing chest pain, shortness of breath, or palpitations after receiving the mRNA vaccine are advised to seek immediate medical attention.

Myocarditis and pericarditis are diagnosed according to the Brighton Collaboration case definitions, ${ }^{11}$ which include clinical symptoms (cardiac or non-specific symptoms), elevated myocardial biomarkers (troponin $\mathrm{T}$, troponin I or CK myocardial band), electrocardiographic, echocardiogram or cardiac magnetic resonance abnormalities and with other alternative aetiologies for symptoms excluded. ${ }^{12}$ Supportive therapy is the mainstay of treatment with cardiac treatment and intervention if needed. Individuals with myocarditis/ pericarditis are advised to rest until symptoms resolved. ${ }^{12}$

Based on the latest scientific data, the benefits of COVID-19 vaccination to the individual, family members, and society outweigh the reported known and potential risks of vaccination (including the possibility of myocarditis and pericarditis) in the current pandemic. ${ }^{13}$ Owing to the recent emergence of SARS-CoV-2 variants with increased transmissibility, higher rates of vaccination will be required to achieve sufficient herd immunity to prevent further community spread, therefore allowing the society to return to normal.

On 30 June 2021, a joint consensus statement was issued by the Hong Kong Paediatric Society, The Hong Kong Society for Paediatric Immunology 
Allergy and Infectious Diseases, the Hong Kong College of Paediatric Nursing, and the Hong Kong Paediatric Nurses Association. ${ }^{14}$ The consensus statement appeals to children and adolescents (aged $\geq 12$ years), parents, adult household members, and child-carers to receive the COVID-19 vaccine for self-protection, and for the physical health and longterm psychosocial development of all children in Hong Kong.

The Centers for Disease Control and Prevention and the Food and Health Bureau of Hong Kong SAR Government endorse the use of Comirnaty vaccine in adolescents mainly because the benefits of vaccination exceed the risks of SARS-CoV-2 infection. ${ }^{15}$ Although there is no alternative vaccine for this age-group currently available, data are being gathered on CoronaVac, which utilises the inactivated virus platform. If this proves safe and effective for children and adolescents, and is approved for use, CoronaVac may be an alternative for this age-group in Hong Kong.

\section{Author contributions}

All authors contributed to the concept or design of the study, acquisition of the data, analysis or interpretation of the data, drafting of the manuscript, and critical revision of the manuscript for important intellectual content. All authors had full access to the data, contributed to the study, approved the final version for publication, and take responsibility for its accuracy and integrity.

\section{Conflicts of interest}

All authors have disclosed no conflicts of interest.

\section{References}

1. Frenck RW, Jr, Klein NP, Kitchin N, et al. Safety, immunogenicity, and efficacy of the BNT162b2 Covid-19 vaccine in adolescents. N Engl J Med 2021;385:239-50.

2. Persons aged 12 to 15 can make reservations to receive BioNTech vaccine from tomorrow. Press release. Hong Kong SAR Government. 10 Jun 2021. Available from: https://www.info.gov.hk/gia/general/202106/10/ P2021061000556.htm. Accessed 22 Jul 2021.

3. Siripanthong B, Nazarian S, Muser D, et al. Recognizing COVID-19-related myocarditis: The possible pathophysiology and proposed guideline for diagnosis and management. Heart Rhythm 2020;17:1463-71.

4. Marshall M, Ferguson ID, Lewis P, et al. Symptomatic acute myocarditis in seven adolescents following PfizerBioNTech COVID-19 vaccination. Pediatrics 2021 Jun 4.
Epub ahead of print.

5. Starekova J, Bluemke DA, Bradham WS, et al. Evaluation for myocarditis in competitive student athletes recovering from coronavirus disease 2019 with cardiac magnetic resonance imaging. JAMA Cardiol 2021;6:945-50.

6. Keinath K, Church T, Kurth B, Hulten E. Myocarditis secondary to smallpox vaccination. BMJ Case Rep 2018;2018:bcr2017223523.

7. Feldstein LR, Tenforde MW, Friedman KG, et al. Characteristics and outcomes of US children and adolescents with multisystem inflammatory syndrome in children (MIS-C) compared with severe acute COVID-19. JAMA 2021;325:1074-87.

8. Whittaker E, Bamford A, Kenny J, et al. Clinical characteristics of 58 children with a pediatric inflammatory multisystem syndrome temporally associated with SARSCoV-2. JAMA 2020;324:259-69.

9. Gargano JW, Wallace M, Hadler SC, et al. Use of mRNA COVID-19 vaccine after reports of myocarditis among vaccine recipients: update from the Advisory Committee on Immunization Practices-United States, June 2021. MMWR Morb Mortal Wkly Rep 2021;70:977-82.

10. Abu Mouch S, Roguin A, Hellou E, et al. Myocarditis following COVID-19 mRNA vaccination. Vaccine 2021;39:3790-3.

11. Myocarditis/pericarditis case definition. 16 July 2021. Available from: https://brightoncollaboration.us/ myocarditis-case-definition-update/. Accessed 22 Jul 2021.

12. Maron BJ, Udelson JE, Bonow RO, et al. Eligibility and disqualification recommendations for competitive athletes with cardiovascular abnormalities: Task Force 3: Hypertrophic cardiomyopathy, arrhythmogenic right ventricular cardiomyopathy and other cardiomyopathies, and myocarditis: a scientific statement from the American Heart Association and American College of Cardiology. Circulation 2015;132:e273-80.

13. Wallace M, Oliver S. COVID-19 mRNA vaccines in adolescents and young adults: benefit-risk discussion. ACIP Meeting. 23 June 2021. Available from: https://www. cdc.gov/vaccines/acip/meetings/downloads/slides-202106/05-COVID-Wallace-508.pdf. Accessed 23 Jul 2021.

14. Joint Statement of The Hong Kong Paediatric Society, The Hong Kong Society for Paediatric Immunology Allergy and Infectious Diseases, Hong Kong College of Paediatric Nursing and Hong Kong Paediatric Nurses Association on occurrence of myocarditis and pericarditis after mRNA COVID-19 vaccination (30 June 2021). Available from: http://www.medicine.org.hk/hkps/statements.php. Accessed 21 Jul 2021.

15. Authorisation of COVID-19 vaccines under the Prevention and Control of Disease (Use of Vaccines) Regulation (Cap.599K). Available from: https://www.fhb.gov.hk/en/ our_work/health/rr3.html. Accessed 7 Jul 2021. 